\title{
Reflets
}

Revue ontaroise d'intervention sociale et communautaire

\section{Bibliographie des articles Aux quatre coins de la province- 1995 à 2003}

Volume 13, numéro 1, 2007

La violence dans tous ses états

URI : https://id.erudit.org/iderudit/016820ar

DOI : https://doi.org/10.7202/016820ar

Aller au sommaire du numéro

Éditeur(s)

Reflets : Revue ontaroise d'intervention sociale et communautaire

ISSN

1203-4576 (imprimé)

1712-8498 (numérique)

Découvrir la revue

Citer ce document

(2007). Bibliographie des articles Aux quatre coins de la province — 1995 à 2003.

Reflets, 13(1), 215-221. https://doi.org/10.7202/016820ar

Tous droits réservés (C) Reflets : Revue ontaroise d'intervention sociale et communautaire, 2007
Ce document est protégé par la loi sur le droit d'auteur. L'utilisation des services d'Érudit (y compris la reproduction) est assujettie à sa politique d'utilisation que vous pouvez consulter en ligne.

https://apropos.erudit.org/fr/usagers/politique-dutilisation/ 


\section{Bibliographie des articles Aux quatre coins de la province- 1995 à 2003}

\section{Volume 1, numéro 1}

CHOLETTE, Chantal et Lise FAUTEUX (1995). «Création d'un centre francophone d'aide et de lutte contre les agressions à caractère sexuel dans Ottawa-Carleton ", printemps, 202-206.

DEROCHE, Francine (1995). "La formation en promotion de la santé en Ontario français ", printemps, 238-244.

FALARDEAU, Marlène (1995). « Le Centre communautaire de santé mentale de Prescott-Russell », printemps, 219-222.

MARION, Sonja (1995). "Implantation de services sociaux scolaires en milieu francophones minoritaire ", printemps, 223-226.

NOLET, Brigitte (1995). «Rapport du FÉFI '94 : Courage et volonté », printemps, 227-232.

PATOINE, Joanne (1995). «Coalition des communautés en santé », printemps, 207-212.

PAYETTE, Lise Andrée (1995). «Des nouvelles du comité francophone provincial de l'Association provinciale de travailleurs sociaux ", printemps, 233-237.

POLIQUIN, Lyne (1995). «Les services à la jeunesse de Hearst », printemps, 216-218.

PRÉMONT, Linda (1995). «En Ontario, on a ce qu'il faut! », printemps, 213-215.

\section{Volume 1, numéro 2}

BERGERON, Josée (1995). «Un programme de formation innovateur et peu coûteux maintenant accessible à tous les francophones de l'Ontario ", automne, 243-245.

DUPONT, Jean-Marc et Johanne NOLIN (1995). «Le mouvement des communautés en santé dans les comtés unis de Prescott-Russell ", automne, 251-245.

GUINDON, Pauline (1995). «Les cuisines collectives de Hearst », automne, 228-231.

LAFRENIÈRE, Ginette et Juliette DENIS (1995). « Le centre de santé communautaire de Sudbury : stratégies et perspectives ", automne, 214-222.

LAROCHELLE, Hélène (1995). "L'Épicerie-bus : un partenariat qui nourrit la communauté ", automne, 237-242.

MALUBUNGI, Mueni (1995). "Coalition contre les rituels de violence faite à la femme ", automne, 223-227. 
PAQUETTE, Jocelyne (1995). «Francophone à Thunder Bay : une activiste sociale parle », automne, 232-236.

THÉBER GE, Sonia (1995). « Franc-O-Forme », automne, 246-250.

\section{Volume 2, numéro 1}

BOULANGER CULLIGAN, Denyse (1996). "À la recherche d'un service en français dans le Nord-ouest de l'Ontario ", printemps, 169-171.

CODERRE, Cécile (1996). "Comité Réseau ", printemps, 181-183.

DUPUIS, Nadya (1996). «L'Union culturelle des Franco-Ontariennes et la violence faite aux femmes et aux enfants ", printemps, 166-168.

FOURNIER, Julie (1996). «Lien : programme explorant le lien entre la violence et la toxicomanie », printemps, 174-177.

OUELLETTE, Rachel (1996). « Mauvais traitements infligés aux aînés », printemps, 184-187.

PENWILL, Kathryn (1996). " Oasis : Centre des femmes », printemps, 178-180.

ROCHELEAU, Monqiue (1996). « La réalité du Nord ", printemps, 188-191.

VELLA, Marie-Josée (1996). "La violence contre les femmes, c'est l'affaire de tout le monde! ", printemps, 172-173.

\section{Volume 2, numéro 2}

BEEVIS, Micheline (1996). «Vieillir à l'aube de l'an $2000 »$, automne, 158-160.

CARRIÈRE, Hélène et Suzanne POIRIER (1996). "Troisième âge en action ", automne, 138143.

DUFRESNE, Sœur Henriette (1996). "L'université du troisième âge (UTA) ", automne, 144-149.

MARTEL, Richard et Carole PINSONNEAULT (1996). "Maintien à domicile francophone ", automne, 150-157.

MASCHERIN, Rita (1996). "Mauvais traitement des personnes âgées : un manuel pratique ", automne, 173-175.

NOLET, Lise et Blanche BÉNÉTEAU (1996). «Options », automne, 167-172.

OUELLET, Rachelle (1996). « Deux projets innovateurs destinés aux aînés francophones d'OttawaCarleton ", automne, 161-166.

\section{Volume 3, numéro 1}

CAMIRAND, Claude (1997). "Résultats du sondage Reflets ", printemps, 168-173.

GRAVEL, Maxim (1997). "Au service de la jeunesse :le programme ontarien l'Avenir ", printemps, 138-142.

LACROIX, Colette B. et Yasmine NEDJAR (1997). "Alphabétisation et lutte contre la pauvreté chez les francophones d'Ottawa-Carleton », printemps, 153-157. 
LANDAU, Linda (1997). " "Au nom de nos enfants ": prendre position pour les enfants de l'Ontario ", printemps, 149-152.

LELIÈVRE, Philippe (1997). «Fiers de nos enfants : des maisons de la famille liées à une approche communautaire globale dans Prescott-Russell ", printemps, 158-161.

MAISON D’AMITIÉ (1997). «Une vidéo sur la violence psychologique », printemps, 167-167.

MALENFANT, Brigitte et Louise DELISLE (1997). « Besoins des francophones à l'égard des services communautaires d'Ottawa-Carleton ", printemps, 143-148.

MARION, Sonja (1997). "Collaborer pour mieux résister aux effets des coupures », printemps, 162-164.

MARTINEAU, Chantal (1997). «Nouveau programme à Grandir Ensemble », printemps, 165166.

\section{Volume 3, numéro 2}

BOUDREAULT, Monique (1997). "Les Sourdes et la communication », automne, 242-249.

CULLIGAN, Denyse (1997). "La Collective lesbienne ", automne, 250-254.

GOSSELIN, Marie-France, CARDINAL, Margot et Blanche PARADIS (1997). «Vous avez dit reconnaissance des acquis? ", automne, 260-268.

LEMIRE, Denise (1997). «Autoroute de l'information : moyen de communication pour les femmes », automne, 278-282.

MONKMAN,Victoria (1997). «Le chemin du retour ", automne, 255-259.

PICARD, Louise et Gisèle BOUR GOIN (1997). «Adolescence et maternité : étude préliminaire ", automne, 269-277.

\section{Volume 4, numéro 1}

BARNES, Derrick (1998). «Le projet de loi 96 et ses répercussions », printemps, 210-216.

BLANCHETTE, Nancy (1998). "Les hauts et les bas d'une entreprise d'insertion biculturelle ", printemps, 205-209.

LAPIERRE PETERS, Myrtha (1998). "À la rencontre de deux solitudes ", printemps, 217-224.

MONETTE, Richard (1998). "Les défis du travail de développement communautaire auprès de la communauté somalienne de la Basse-Ville d'Ottawa ", printemps, 194-199.

MUAMBA, Marie-Claude (1998). «Barrières linguistiques et intégration des nouveaux immigrants francophones à Toronto ", printemps, 182-187.

NDUWIMANA, Mathilde (1998). «Entre le marteau et l'enclume : le vécu des intervenantes et intervenants interculturels ", printemps, 176-181.

SIMARD, Anne (1998). "Les intervenants et les organismes francophones en période de bouleversement ", printemps, 188-193.

WEINTRAUB, Lisa (1998). « En toute honnêteté », printemps, 200-204. 


\section{Volume 4, numéro 2}

Centre FORA (1998). «Le Centre franco-ontarien de ressources en alphabétisation : Centre FORA », automne, 214-216.

DUGAS, Carmelle (1998). « Maison La Paix : c'et quoi? », automne, 191-194.

GAGNÉ, Hélène (1998). "La toute nouvelle Fondation de neurotraumatologie », automne, 186-190.

GOUNANE, Smail (1998). «Le pouvoir des mots », automne, 207-208.

GROULX, Carole (1998). "Le système de coordination des services, un modèle à suivre ", automne, 201-206.

POIRIER, Alain et Monique MORIN-LAVIGNE (1998). «Le P'tit Bonheur : un programme du Patro d'Ottawa pour les personnes ayant un handicap intellectuel ", automne, 212-213.

RESO (1998). «RESO, le regroupement des parents et amis des enfants sourds et malentendants franco-ontariens ", automne, 209-211.

THOMAS, Noël (1998). "L'Internet : nouvelle ressource ou nouvelle barrière? », automne, 195-200.

\section{Volume 5, numéro 1}

DENNIE, Donald (1999). "Je refuse de laisser mourir mon village », printemps, 152-158.

DUPONT, Natalie (1999). "L'engagement de la Caisse populaire dans le développement de la communauté de Kapuskasing ", printemps, 168-175.

KIHUMBI, Mufaume (1999). "La Maison Verte : un cas réussi de développement économique communautaire ", printemps, 176-183.

LEMIEUX, Jean-Gilles(1999). "Les modes d'organisation du travail dans une entreprise de développement économique communautaire : le cas de la Coopérative Boréal ", printemps, 159-167.

LEMIRE, Denise (1999). «Une recherche-action du RÉSAFF Les travailleuses autonomes de l'Est de l'Ontario : enjeux et défis vers l'égalité ", automne, 184-190.

\section{Volume 5, numéro 2}

BARIBEAU, Jocelyne et Zehira HOUFANI, (1999). «Alphabétisation familiale », automne, 258262.

FRASER, Colette (1999). "Le cours d'été en promotion de la santé, session 1999 ", automne, 270-278.

NOLET, Lise (1999). "Santé des organismes communautaires inc. », automne, 285-288.

PLANTE, Diane (1999). "Le RIFSSSO - un modèle de coopération ", automne, 279-284.

RACINE, Guy (1999). «Le Programme régional des soins palliatifs de Sudbury : une réussite à souligner et à protéger", automne, 268-269.

THOMAS, Noël (1999). "Le Réseau de professionnels à distance : un modèle de prestation de services ", automne, 263-267. 


\section{Volume 6, numéro 1}

CHAMPION, Natalie et Nancy BARREIRA (2000). "La promotion de l'activité physique : contrer la sédentarité », printemps, 220-225.

DUPRAS, Cilvy (2000). «Intervention et crise suicidaire », printemps, 197-207.

GARCEAU, Marie-Luce (2000). «Fiches atouts! Trousse de formation sur la violence conjugale et l'agression sexuelle contre les femmes ", printemps, 216-219.

GÉROME, Marie-Hélène (2000). "La Marche mondiale des femmes en l'an 2000 en Ontario français ", printemps, 192-196.

LEMIEUX, Micheline (2000). "Développement d'un programme bénévole pour les personnes psychiatrisées en crise", printemps, 208-215.

PLANTE, Jacqueline (2000). « Mon corps, mon trésor », printemps, 226-229.

\section{Volume 6, numéro 2}

ASEZO, Myriame Odyssée Amenanka (2000). «S'alphabétiser en français dans un milieu anglophone : défi à relever pour l'intégration des francophones analphabètes sur le marché de l'emploi de Toronto ", automne, 221-225.

BISSON, Marc (2000). Entrevue recueillie par Nérée St-Amand, «Jardins communautaires et mobilisation sociale à Cornwall ", automne, 203-216.

GÉROME, Marie-Hélène (2000). «Une Marche des femmes en Ontario français en solidarité avec toutes les femmes du monde! ", automne, 217-220.

LABRÈCHE, Carole (2000). "Le vent du large », automne, 202.

PARROT, Claire (2000). "La santé sexuelle des jeunes femmes afro-francophones de Toronto ", automne, 226-230.

TURRITTIN, Jane (2000). "Le Cinéma Africain : au service de la diaspora africaine et de la communauté torontoise cosmopolitaine ", automne, 231-234.

TURRITTIN, Jane (2000). «Le Regroupement des jeunes filles francophones de Toronto : œuvrer pour l'épanouissement de la jeune fille francophone de Toronto », automne, 235-240.

\section{Volume 7, numéro 1}

GÉROME, Marie-Hélène (2001). "Aperçu des comportements de santé en lien avec l'image corporelle des adolescentes francophones vivant en milieu minoritaire ", printemps, 194-199.

JOANISSE, Julie (2001). "Un Témoignage - le profil de Philippe », printemps, 200-204.

\section{Volume 7, numéro 2}

GARCEAU, Marie-Luce (2001). «Pour exprimer son histoire en français », automne, 145-150.

LAFLEUR, Marie-Paule (2001). "L'Association ontarienne des Sociétés de l'aide à l'enfance (AOSAE), connaissez-vous? ", automne, 141-144. 
MASCHERIN, Rita (2001). "L'ATTSO : la voix des travailleuses et travailleurs sociaux en Ontario ", automne, 132-140.

MUNROE,Viviane (2001). "Perceptions de la criminalité et évaluation du niveau de sécurité : une étude qualitative dans un quartier sudburois ", automne, 151-156.

\section{Volume 8, numéro 1}

GOULET, Anik (2002). «La pratique de la physiothérapie en milieu minoritaire : mon expérience en tant que francophone à l'Hôpital de Hamilton ", printemps, 164-168.

JOANISSE, Dr. John (2002). "Le rôle de l'hôpital communautaire Montfort comme pilier francophone de formation ", printemps, 169-172.

OUELLETTE, Rachel et Roland LECOMTE (2002). «Population vieillissante oblige : formation à l'intention des intervenants en santé et services sociaux », printemps, 158-163.

RAJROOP, Janet (2002). Entrevue recueillie par Lynn Casimiro, « Modèle d'apport de services en santé communautaires aux francophones du Sud de l'Ontario ", printemps, 173-176.

\section{Volume 9, numéro 1}

ANDREW, Caroline et Linda CARDINAL (2003). « Un pouvoir à partager :les femmes francophones de l'Ontario dans la politique municipale et scolaire, état de la situation ", printemps, 212-215.

BOUCHARD, Lyne (2003). "Les principes d'intervention à la Table féministe de concertation provinciale de l'Ontario ", printemps, 216-221.

DENIS, Ann et Michèle OLIVIER (2003). "L'utilisation des technologies d'information et de communication chez les femmes francophones en situation minoritaire au Canada ", printemps, 222-230.

DÉRY, Élaine (2003). «Le secteur “Femmes” de l'Entente Canada-communauté-Ontario : des priorités pour l'avenir », printemps, 231-235. 